\title{
Testosterone Propionate Exacerbates the Deficits of Nigrostriatal Dopaminergic System and Downregulates Nrf2 Expression in Reserpine-Treated Aged Male Rats
}

Rui Cui ${ }^{1,2 \dagger}$, Yunxiao Kang ${ }^{1 \dagger}$, Li Wang ${ }^{1}$, Shuangcheng $\mathrm{Li}^{2}$, Xiaoming $\mathrm{Ji}^{1}$, Wensheng Yan ${ }^{1}$, Guoliang Zhang ${ }^{1,2}$, Huixian Cui ${ }^{2}$ and Geming Shi ${ }^{1 *}$

${ }^{1}$ Department of Neurobiology, Hebei Medical University, Shijiazhuang, China, ${ }^{2}$ Department of Human Anatomy, Hebei Medical University, Shijiazhuang, China

There is a controversy over the effects of testosterone supplements on dopaminergic function. Both neuroprotective and toxic effects of testosterone supplements are reported. The status of oxidative stress seems to explain the neuroprotective or toxic properties of testosterone. To determine the efficacy of testosterone supplements in different status of oxidative stress, the present studies analyzed the dopamine (DA)-related behaviors and neurochemical indices, as well as markers of nigrostriatal dopaminergic (NSDA) system in reserpine-treated aged male rats followed by testosterone propionate (TP) supplements. The status of oxidative stress of experimental animals was evaluated by analyzing oxidative stress parameters and nuclear factor erythroid 2-related factor 2 (Nrf2)-antioxidant response element (ARE) signaling pathway in substantia nigra (SN). Consistent with our previous studies, TP supplements to 21-month old aged male rats had the beneficial effects on NSDA system and DA-related behaviors and enhanced the antioxidative capabilities in SN. However, the beneficial effects of TP supplements on NSDA system and DA-related behaviors in aged male rats were reversed by reserpine pretreatment to them. Reserpine treatment induced the severe oxidative stress and reduced the expressions of Nrf2, heme oxygenase-1 ( $\mathrm{HO}-1)$ and $\mathrm{NAD}(\mathrm{P}) \mathrm{H}$ :quinone oxidoreductase-1 (NQO1) in the $\mathrm{SN}$ of aged male rats. The TP supplements to reserpine-pretreated aged male rats exacerbated the defects in NSDA system and DA-related behaviors, aggravated oxidative damages and downregulated the expression of Nrf2, $\mathrm{HO}-1$ and NQO1 in the SN. These results suggested that the efficacy of TP supplements on impaired NSDA system was related to the status of oxidative stress in experimental rats.

Keywords: testosterone propionate, reserpine, nigrostriatal dopaminergic system, oxidative stress, Nrf2-ARE, aged male rats
Cui R, Kang Y, Wang L, Li S, Ji X, Yan W, Zhang G, Cui H and Shi G

(2017) Testosterone Propionate Exacerbates the Deficits of Nigrostriatal Dopaminergic System and Downregulates Nif2 Expression in Reserpine-Treated Aged

Male Rats.

Front. Aging Neurosci. 9:172. doi: 10.3389/fnagi.2017.00172
Abbreviations: $\mathrm{ARE}$, antioxidant response element; $\mathrm{CPu}$, caudate putamen; $\mathrm{DA}$, dopamine; $\mathrm{DAT}$, dopamine transporter; DOPAC, 3,4-dihydroxyphenylacetic acid; GSH, glutathione; HO-1, heme oxygenase-1; HVA, homovanillic acid; MDA, malondialdehyde; NQO1, NAD(P)H:quinone oxidoreductase-1; Nrf2, nuclear factor erythroid 2-related factor 2; NSDA, nigrostriatal dopaminergic; SN, substantia nigra; TH, tyrosine hydroxylase; TP, testosterone propionate. 


\section{INTRODUCTION}

Oxidative stress is involved in the progression of aging and aging-related dysfunction (Hamilton et al., 2001; Kregel and Zhang, 2007; Wang and Michaelis, 2010), and induces the progressive and irreversible oxidative damages during dopaminergic neurodegeneration (Chen et al., 2008; Hwang, 2013). Regulation of status of oxidative stress in brain might be an effective strategy against the aging-related dopaminergic neurodegeneration (Hwang, 2013; Villar-Cheda et al., 2014). The nuclear factor erythroid 2-related factor 2 (Nrf2), as a transcription factor, controls the basal and inducible expression of an array of antioxidant and detoxification enzymes by binding antioxidant response element (ARE; Carmona-Aparicio et al., 2015; Miller et al., 2015). Activated Nrf2 induces a series of antioxidant genes to protect cells against oxidative stress (Dou et al., 2016). Thus, the activation of Nrf2 is an important approach to attenuate oxidative stress (van Muiswinkel and Kuiperij, 2005; Dou et al., 2016).

Androgens can manipulate the behaviors of organisms (Frye et al., 2008; Zhang et al., 2011) by influencing central dopaminergic activity (Abreu et al., 1988; Kindlundh et al., 2004; de Souza Silva et al., 2009). The supplements of testosterone improve motor symptoms in Parkinson's disease (Mitchell et al., 2006) and motor behavioral defects in castrated male rats and aged male rats (Zhang et al., 2011; Cui et al., 2012), and enhance dopaminergic activity (Abreu et al., 1988; Zhang et al., 2016). The enhanced dopaminergic activity in aged rats by testosterone seems related to the ameliorated status of oxidative stress (Zhang et al., 2016). Testosterone treatment decreases the vulnerability of neurons of neonatal rats to oxidative stress in vitro (Ahlbom et al., 1999) and suppresses orchiectomy-induced oxidative damage and neuronal morphological changes in the hippocampus of adult rats (Meydan et al., 2010). Testosterone supplements to aged male rats activate the Nrf2-ARE pathway and strengthen the antioxidant capability (Zhang et al., 2013, 2016). Androgens exert neuroprotective action against oxidative stress (Ahlbom et al., 1999; Meydan et al., 2010).

Considering the influence of androgens upon the dopaminergic activity and behaviors (Frye et al., 2008; de Souza Silva et al., 2009), as well as the lower testosterone levels in aged men (Hollander et al., 2003), testosterone replacement might be regarded as a therapeutic treatment in aged men against aging-related degenerative disorders (Tenover, 1992; Pike et al., 2009). However, the efficacy of testosterone replacement in aged men is controversial (Okun et al., 2002; Cunningham and Toma, 2011) and both suppressive effects (Dluzen and Ramirez, 1989; Hernandez et al., 1994) and facilitating influences (Abreu et al., 1988; Martínez-Sanchis et al., 2002; de Souza Silva et al., 2009) of testosterone propionate (TP) supplements on the dopaminergic activity are also reported in animal experiments. In addition to the organisms studied (Frye et al., 2010; Borbélyová et al., 2016) and the treatment regimen of androgens (Estrada et al., 2006; Spritzer et al., 2011), a critical factor might determine the efficacy of testosterone supplements. Based on the effects of oxidative stress on aging-related dopaminergic neurodegeneration (Chen et al., 2008; Hwang, 2013), the status of oxidative stress in organisms might be the candidate for the discrepancy when androgens are supplemented.

Reserpine, a blocker of the vesicular monoamine transporter, is a powerful oxidant (Metzger et al., 2002). It increases cytoplasmic dopamine (DA) by preventing the storage of DA in dopaminergic synaptic vesicles. The DA not stored in synaptic vesicles is subjected to degradation either by autoxidation or by oxidative deamination catalyzed by monoamino oxidase (MAO; Lohr, 1991). As a result, the increased cytoplasmic DA causes an acceleration of DA catabolism by MAO as well as an elevated autoxidation of DA, which results in the overproduced reactive species involved in the degenerative processes (Paris et al., 2001; Fuentes et al., 2007) and the alteration in the status of the oxidative stress of dopaminergic nerve terminals (Bilska et al., 2007). Therefore, to investigate the potential influence of oxidative stress on testosterone supplements, the DA-related behaviors and neurochemical indices, as well as markers of nigrostriatal dopaminergic (NSDA) system were analyzed in reserpine-treated aged male rats followed by TP treatment. Meanwhile, oxidative stress parameters and Nrf2-ARE pathway in NSDA system were examined to evaluate the status of oxidative stress of experimental animal models.

\section{MATERIALS AND METHODS}

\section{Animals and Housing}

Fifty male Wistar rats supplied by the Experimental Animal Center of Hebei Medical University were housed in an air-conditioned room $\left(22 \pm 3^{\circ} \mathrm{C}\right)$ on a $12 \mathrm{~h}$ light-dark cycle (lights on 6:00 AM). Food and water were available ad libitum. All of the experimental procedures were conducted in accordance with the rules in the Guidelines for the Care and Use of Mammals in Neuroscience and Behavioral Research and were approved by the Committee of Ethics on Animal Experiments at Hebei Medical University.

\section{Testosterone Propionate Supplements}

All of the rats were randomly divided into the following five groups: 6-month-old group (6Mon, $n=10$ ), 22-monthold group (22Mon, $n=10$ ), 22-month-old TP group (22MonTP, $n=10)$, 22-month-old reserpine group (22MonR, $n=10$ ) and 22-month-old reserpine-treated TP group (22MonR-TP, $n=10$ ). For $22 \mathrm{MonR}$ and $22 \mathrm{MonR}-\mathrm{TP}$, the rats at the age of 21 months received a single subcutaneous injection of reserpine (5 mg/kg; CAS: 50-55-5, Tokyo Chemical industry CO., Japan; Bilska et al., 2007). The rats in 22Mon and 22Mon-TP got same treatment with $0.1 \%$ glacial acetic acid solution $(1 \mathrm{ml} / \mathrm{kg})$ as vehicle instead of reserpine. Forty-eight hours after injection, the rats in 22Mon-TP and 22MonR-TP received daily subcutaneous TP injection (2 mg/kg; Cat. NO.: 35, 805, SERVA Electrophoresis, Germany; Martínez-Sanchis et al., 2002; Chen et al., 2003), which was continued for 4 weeks (28 days). The rats in 22Mon and $22 \mathrm{MonR}$ received the same treatment using sesame oil (0.1 ml/kg; MKBH4400V, S3547-250ML, Sigma, USA) as a vehicle. 


\section{Open-Field Test}

All of the rats in five groups were used for open-field test. Each rat was placed in an open field chamber $(100 \times 100 \times 40 \mathrm{~cm})$ according to the procedure used in our previous study (Zhang et al., 2011; Cui et al., 2012). The bottom of open field chamber was lined into 25 squares $(20 \times 20 \mathrm{~cm})$. Every square further consisted of 400 small grills $(1 \times 1 \mathrm{~cm})$. At the 27 th day and 28th day of TP treatment, open-field test was performed for all five groups. Open-field behavior was recorded for $5 \mathrm{~min}$. Three behavioral parameters were observed in the study. They are vertical activity, horizontal activity and total path length, which respectively refer to the total number of erect posture, the total number of square crossings and the total length of crossings of the rat in whole test period. Averaged two measurements at the 27th day and 28th day were made a mean alone to present as an individual result for one rat. The averaged amount of the two measurements of one rat at the 27th day and 28th day was summarized in each group of five groups for later analysis.

\section{Adhesive Removal Test}

Adhesive removal test was performed in home cage as described in our previous study (Cui et al., 2012; Wang et al., 2016) according to Schallert et al. (1982). After adaptive training (5 days, 1 trial per day), all of the rats in each group were tested on the day when last-open-field test ended. Latency to remove stimulus of adhesive paper (Avery) from each side of snout and each forepaw was documented. The first of total three trials was used in data analysis (Schallert et al., 1982).

\section{Sample Preparation}

Following the behavioral tests, the rats in each group were sacrificed by decapitation. The brains were removed quickly. The tissue blocks containing substantia nigra ( $\mathrm{SN}$; between $3.00 \mathrm{~mm}$ and $4.08 \mathrm{~mm}$ rostral to the interaural axis) or caudateputamen $(\mathrm{CPu}$; between $10.08 \mathrm{~mm}$ and $8.64 \mathrm{~mm}$ rostral to the interaural axis; Paxinos and Watson, 2007) were dissected on ice-cold plate, using a scalpel for ophthalmic surgery and a stereomicroscopy. The $\mathrm{CPu}$ tissue blocks from five rats in each group were processed for analyzing tyrosine hydroxylase (TH) and DA transporter (DAT) at protein level, and those from the other five rats were prepared for detecting DA and its metabolite contents. The unilateral SN tissue blocks from five rats in each group were chosen for analyzing $\mathrm{TH}$ and DAT at protein level or used for assaying malondialdehyde (MDA) and reduced glutathione (GSH) contents. The unilateral SN tissue blocks from other five rats in each group were processed for analyzing Nrf2, heme oxygenase-1 (HO-1) and $\mathrm{NAD}(\mathrm{P}) \mathrm{H}$ :quinone oxidoreductase-1 (NQO1) at protein level or for measuring Nrf2, HO-1 and NQO1, as well as TH and DAT at mRNA level. The tissue samples for the protein and mRNA detection were frozen in liquid nitrogen and stored at $-80^{\circ} \mathrm{C}$ for subsequent analysis, and the samples for MDA and GSH as well as DA and its metabolite assay were processed immediately after brain dissection.

\section{LC-MS/MS Assay}

The tissue blocks of $\mathrm{CPu}$ were weighed and homogenized in $80 \%$ acetonitrile containing $0.1 \%$ formic acid $(5 \mu \mathrm{l} / \mathrm{mg})$. The homogenates were centrifuged at $14,000 \mathrm{rpm}$ for $10 \mathrm{~min}$ at $4^{\circ} \mathrm{C}$. The supernatants were collected and stored at $-80^{\circ} \mathrm{C}$. DA, 3,4-dihydroxyphenylacetic acid (DOPAC) and homovanillic acid (HVA) in tissue blocks were determined by LC-MS/MS (Sun et al., 2013). The LC separation was performed on Agilent 1200 LC system (Agilent, Santa Clara, CA, USA) using a Synergi Fusion-RP C18 column $(50 \mathrm{~mm} \times 3.0 \mathrm{~mm}, 4 \mu \mathrm{m})$ provided by Phenomenex. The MS/MS detection was carried out using a 3200 QTRAP $^{\text {TM }}$ LC-MS/MS system (Applied Biosystems, Foster City, CA, USA). The multiple-reaction monitoring mode was used for the quantification.

\section{Quantitative Real-Time PCR Analysis}

Total RNA $(2 \mu \mathrm{g})$ from SN tissue block was subjected to reverse transcription using random primer to obtain the first-strand cDNA template. Quantitative real-time PCR was performed with $0.8 \mu \mathrm{l}$ cDNA (diluted 1:10), $2 \mu l$ (10 pmoles each) specific primers and $2 \times$ GoTaq $^{\circledR}$ Green Master Mix (Promega, Madison, WI, USA) with a final volume of $20 \mu \mathrm{l}$. PCR was performed as follows: an initial heat activation step at $95^{\circ} \mathrm{C}$ for $10 \mathrm{~min}$, followed by 40 cycles with a denaturation step at $95^{\circ} \mathrm{C}$ for $15 \mathrm{~s}$, an annealing step for $20 \mathrm{~s}$, and an extension step at $72^{\circ} \mathrm{C}$ for $27 \mathrm{~s}$. The products were analyzed by melting curve to confirm the specificity of amplification. Expression of $\mathrm{TH}$, DAT, Nrf2, HO-1 and NQO1 genes was analyzed using glyceraldehyde-3-phosphate dehydrogenase (GAPDH) as an internal control. The relative quantification was calculated using the $2^{-\Delta \Delta \mathrm{ct}}$ method. The sets of primers were as follows: TH (5'-GCTTCTCTGACCAGGTGTATCG-3' ${ }^{\prime}$ and 5'-GCAATCTCTTCCGCTGTGTAT-3'; annealing temperature: $\left.60^{\circ} \mathrm{C}\right)$, DAT $\left(5^{\prime}\right.$-ACTCTGTGAGGCATCTGTGTG- $3^{\prime}$ and $5^{\prime}-$ TGTAACTGGAGAAGGCAATCAG- $3^{\prime}$; annealing temperature: $\left.59^{\circ} \mathrm{C}\right), \quad \mathrm{Nrf} 2 \quad\left(5^{\prime}\right.$-GACCTAAAGCACAGCCAACACAT- $3^{\prime}$ and $\quad 5^{\prime}$-CTCAATCGGCTTGAATGTTTGTC-3'; annealing temperature: $59^{\circ} \mathrm{C}$; Wang et al., 2014), HO-1 (5'-TGTCCC AGGATTTGTCCGAG-3' and $5^{\prime}$-ACTGGGTTCTGCTTGTT TCGCT-3'; annealing temperature: $60^{\circ} \mathrm{C}$; Wang et al., 2014), NQO1 (5'-GGGGACATGAACGTCATTCTCT- $3^{\prime}$ and $5^{\prime}$-AG TGGTGACTCCTCCCAGACAG-3'; annealing temperature: $62^{\circ} \mathrm{C}$; Wang et al., 2014), GAPDH (5'-TGAACGGGAAGCTC ACTG- $3^{\prime}$ and $5^{\prime}$-GCTTCACCACCTTCTTGATG-3'; annealing temperature: $58^{\circ} \mathrm{C}$; Wang et al., 2014). Primer specificity was confirmed by blasting the primer sequence against genomic databases available at NCBI.

\section{Western Blot Analysis}

The tissue blocks for detection of $\mathrm{TH}$ and DAT protein levels were homogenized in Radio Immuno Precipitation Assay (RIPA) buffer containing 1\% Triton X-100, 0.1\% SDS, $0.5 \%$ sodium deoxycholate and protease inhibitors $(100 \mu \mathrm{g} / \mathrm{ml}$ phenylmethanesulfonyl fluoride, $30 \mu \mathrm{g} / \mathrm{ml}$ aprotinin, $1 \mathrm{mM}$ sodium orthovanadate), and then sonicated for $4 \times 10 \mathrm{~s}$. After centrifugation at $12,000 \times g$ for $20 \mathrm{~min}$ at $4^{\circ} \mathrm{C}$, the 
supernatant was collected and centrifuged again as described above. The final resulting supernatant was stored at $-80^{\circ} \mathrm{C}$ until use. Samples from SN $(80 \mu \mathrm{g})$ or $\mathrm{CPu}(50 \mu \mathrm{g})$ were diluted with $2 \times$ sample buffer ( $50 \mathrm{mM}$ Tris, $\mathrm{pH} 6.8,2 \%$ SDS, $10 \%$ glycerol, $0.1 \%$ bromophenol blue, $5 \% \beta$-mercaptoethanol) and heated for $5 \mathrm{~min}$ at $95^{\circ} \mathrm{C}$ before SDS-PAGE on a $10 \%$ gel, and subsequently transferred to PVDF membrane (Millipore). After incubated for $2 \mathrm{~h}$ with $5 \%$ non-fat dry milk in Tris-buffered saline (TBS) containing 0.05\% Tween 20 (TBST; 20 mM Tris-Cl, $137 \mathrm{mM} \mathrm{NaCl}, 0.1 \%$ Tween 20, $\mathrm{pH}$ 7.6) at room temperature, the membrane was rinsed in three changes of TBST and then incubated overnight with mouse anti-TH monoclonal antibody (Sigma, T2928, 1:10,000) or rabbit anti-DAT polyclonal antibody (Millipore, Temecula, CA, USA; AB2231, 1:4000) at $4^{\circ} \mathrm{C}$. After three washes, the membrane was incubated for $1 \mathrm{~h}$ in IRDye ${ }^{\circledR} 800$-conjugated goat anti-mouse second antibody (1:3000, Rockland) or goat anti-rabbit second antibody (1:3000, Rockland) at room temperature. The membrane was scanned by Odyssey infrared scanner (LI-COR Biosciences). Following stripping, each PVDF membrane was subsequently immunoblotted with mouse anti- $\beta$-actin monoclonal antibody (1:6000, Santa Cruz Biotechnology). The labeling densities for $\mathrm{TH}$ or DAT were compared with those of $\beta$-actin and analyzed by Gel-Pro Analyser Analysis software (Media Cybernetics).

The tissue blocks for detection of Nrf2, HO-1 and NQO1 protein levels were homogenized in an ice-cold lysis buffer (10 mmol/L HEPES, $\mathrm{pH} 7.9,10 \mathrm{mmol} / \mathrm{L} \mathrm{KCl}$, $0.1 \mathrm{mmol} / \mathrm{L}$ EDTA, $1 \mathrm{mmol} / \mathrm{L}$ DTT, $0.1 \mathrm{mmol} / \mathrm{L}$ EGTA) including protease inhibitors $(100 \mu \mathrm{g} / \mathrm{ml}$ phenylmethanesulfonyl fluoride, $30 \mu \mathrm{g} / \mathrm{ml}$ aprotinin, $1 \mathrm{mM}$ sodium orthovanadate) for 15 min. After adding NP-40, the homogenate was centrifuged at $10,000 \mathrm{rpm}$ at $4^{\circ} \mathrm{C}$ for $3 \mathrm{~min}$, and the supernatant was collected as cytoplasmic protein for HO-1 and NQO1. The pellets were homogenized in an ice-cold lysis buffer (20 mmol/L HEPES, pH 7.9, $400 \mathrm{mmol} / \mathrm{L} \mathrm{NaCl}, 1 \mathrm{mmol} / \mathrm{L}$ EDTA, $0.1 \mathrm{mmol} / \mathrm{L}$ EGTA) for $15 \mathrm{~min}$. After centrifugation at $12,000 \mathrm{rpm}$ for $10 \mathrm{~min}$ at $4^{\circ} \mathrm{C}$, the supernatant was collected. Phenylmethanesulfonyl fluoride was added to the supernatant with the final concentration of $1 \mathrm{mmol} / \mathrm{L}$ as the nuclear protein for Nrf2. Samples from SN $(80 \mu \mathrm{g})$ were separated by SDS-PAGE and transferred to PVDF membrane. The membrane was blocked with 5\% skimmed milk for $1 \mathrm{~h}$, and then was probed with polyclonal rabbit anti-Nrf2 antibody (Abcam, Cambridge, MA, USA, 1:500), polyclonal rabbit anti-HO-1 antibody (Abcam, Cambridge, MA, USA, 1:200) or polyclonal rabbit anti-NQO1 antibody (Abcam, Cambridge, MA, USA, 1:200) overnight at $4^{\circ} \mathrm{C}$. After three washes, IRDye ${ }^{\circledR} 800$-conjugated goat anti-rabbit second antibody (1:3000, Rockland) incubated with membranes for $1 \mathrm{~h}$ at room temperature. The membrane was scanned by Odyssey infrared scanner (LI-COR Biosciences). The densitometry values were normalized with respect to the values of anti-histone $3(\mathrm{H} 3$, bioWORLD, Dublin, OH, USA, 1:1000) for Nrf2 or anti- $\beta$-actin for HO-1 and NQO-1 immunoreactivity. The labeling densities were analyzed by Gel-Pro Analyser Analysis software (Media Cybernetics).

\section{MDA and GSH Assay}

The tissue blocks of SN were homogenized with 10 times $(\mathrm{w} / \mathrm{v})$ ice-cold $0.1 \mathrm{M}$ phosphate buffer (PB) at $\mathrm{pH}$ 7.4. The homogenates were used to assess reduced GSH and lipid peroxidation product. The levels of GSH and MDA were measured spectrophotometrically using detection kits, following the manufacturer's instruction (GSH: 016,835; MDA: 022,446, Nanjing Jiancheng Bioengineering Institute, China).

\section{Serum Testosterone Assay}

Samples of trunk blood were collected from the rats used for western blot analysis after decapitation and sat in open microfuge tubes at room temperature for about $30 \mathrm{~min}$ to allow the blood to coagulate. Serum samples were prepared by centrifugation and stored at $-80^{\circ} \mathrm{C}$ until assay. Serum testosterone levels were determined by radioimmunoassay using testosterone radioimmunoassay kit (Tianjin Nine Tripods Medical and Bioengineering Co., Ltd. China) according to the protocol of the kit.

\section{Statistical Analysis}

All of the data were described as mean \pm SD. The tests of normality (Kolmogorov-Smirnov test) and homogeneity variance (Levene's test) were applied to all of the data. If both normal distribution $(p>0.1)$ and homogeneity of variance $(p>0.1)$ were found, the parametric test was performed by one-way analysis of variance (one-way ANOVA) followed by a Student-Newman-Keuls (SNK) post hoc test for multiple comparisons. Otherwise, nonparametric statistics were done by a Kruskal-Wallis test, where $p<0.05$, post hoc comparisons between groups were performed using the Mann-Whitney $U$ test. Differences were considered to be significant when $p$ values were less than 0.05 . However, for nonparametric statistics using Kruskal-Wallis test $(p<0.05)$, the Mann-Whitney $U$ test was done followed by Bonferroni correction. Bonferroni correction was used for multiple comparisons, and the predefined significance level $(p<0.05)$ was reset at $p<0.0125$ after Bonferroni correction.

\section{RESULTS}

\section{Serum Testosterone Concentration}

The serum testosterone was measured to determine the levels of testosterone in experimental rats. Group differences in the levels of serum testosterone were found among $6 \mathrm{Mon}, 22 \mathrm{Mon}, 22 \mathrm{Mon}-$ $\mathrm{TP}, 22 \mathrm{MonR}$ and $22 \mathrm{MonR}-\mathrm{TP}$ rats $\left(F_{(4,20)}=94.247, p<0.01\right)$. The levels of serum testosterone were lower in $22 \mathrm{Mon}$ rats $(1.77 \pm 0.70 \mathrm{ng} / \mathrm{ml})$ than that in 6 Mon rats $(4.59 \pm 0.81 \mathrm{ng} / \mathrm{ml}$; $p<0.01)$. Supplements of TP for 4 weeks resulted in higher levels of serum testosterone in 22Mon-TP rats $(11.64 \pm 1.74 \mathrm{ng} / \mathrm{ml})$ than that in $22 \mathrm{Mon}$ rats $(p<0.01)$. No significant difference existed in the levels of serum testosterone between 22Mon and $22 \mathrm{MonR}$ rats, as well as between $22 \mathrm{Mon}-\mathrm{TP}$ and $22 \mathrm{MonR}-\mathrm{TP}$ rats. 


\section{Behavior Tests}

To observe the effects of TP supplements on reserpine-treated aged male rats, DA-related behaviors were analyzed by open-field test and adhesive removal test.

\section{Open-Field Behavior}

The motor behaviors of experimental rats were observed by detecting vertical activity, horizontal activity and total path length in open-field test. Analysis to the motor behaviors revealed group differences among 6Mon, 22Mon, 22Mon-TP, 22MonR and 22MonR-TP rats in the amount of vertical activity (Figure 1A, $\chi^{2}=39.169, p<0.001$ ) and horizontal activity (Figure 1B, $\chi^{2}=38.797, p<0.001$ ), as well as total path length (Figure 1C, $\chi^{2}=41.604, p<0.001$ ). The post hoc test found that the amount of vertical activity and horizontal activity, as well as total path length significantly decreased in 22Mon rats compared with 6Mon rats $(p<0.001)$. The amount of vertical activity $(p=0.012)$ and total path length $(p=0.01)$ significantly increased in 22Mon-TP rats compared with 22Mon rats. Marked reduction in the amount of vertical activity $(p=0.011)$ and horizontal activity $(p=0.012)$, as well as total path length $(p=0.01)$ was detected in $22 \mathrm{MonR}$ rats compared with $22 \mathrm{Mon}$ rats. Supplements of TP reduced the total path length in reserpinetreated aged male rats $(p=0.01)$.

\section{Adhesive Removal Test}

Adhesive removal test demonstrated group differences among $6 \mathrm{Mon}, 22 \mathrm{Mon}, 22 \mathrm{Mon}-\mathrm{TP}, 22 \mathrm{MonR}$ and 22MonR-TP rats in the latency to remove snout stimuli (Figure 2A, left side of snout: $\chi^{2}=45.499, p<0.001$; right side of snout: $\chi^{2}=45.258$, $p<0.001$ ) and forepaw stimuli (Figure 2B, left forepaw: $\chi^{2}=46.324, p<0.001$; right forepaw: $\chi^{2}=46.874, p<0.001$ ). The post hoc test revealed the longer latency to remove the stimuli from each side of snout and each forepaw in 22Mon rats than in 6 Mon rats $(p<0.001)$. In 22Mon-TP rats, the latency to remove the stimuli from each side of snout and each forepaw significantly decreased compared with 22Mon rats (left side of snout: $p=0.002$; right side of snout: $p=0.005$; left forepaw: $p<0.001$; right forepaw: $p<0.001)$. The significant increase in the latency to remove the stimuli from snout and forepaws was observed in 22MonR rats compared with 22Mon rats (left side of snout: $p=0.001$; right side of snout: $p=0.001$; left forepaw: $p=0.001$; right forepaw: $p<0.001$ ) and in 22MonR-TP rats compared with $22 \mathrm{MonR}$ rats $(p<0.001)$. Supplements of TP increased the latency to remove stimuli in reserpine-treated aged male rats.

\section{Nigrostriatal Dopaminergic System}

The markers of NSDA system were analyzed to investigate the effects of TP supplements on the impaired NSDA system in reserpine-treated aged male rats.

\section{DA and its Metabolites}

Group differences were disclosed among 6Mon, 22Mon, 22Mon$\mathrm{TP}$, 22MonR and 22MonR-TP rats in DA (Figure $\mathbf{3 A}$, $\chi^{2}=22.228, p<0.001$ ), DOPAC (Figure 3B, $F_{(4,20)}=69.558$, $p<0.01$ ) and HVA (Figure 3C, $F_{(4,20)}=40.181, p<0.01$ ) content in CPu. Post hoc test showed that DA, DOPAC and HVA significantly reduced in 22Mon rats compared with $6 \mathrm{Mon}$ rats (DA: $p=0.009$; DOPAC: $p<0.01$; HVA: $p<0.01$ ), and increased in 22Mon-TP rats compared with 22Mon rats (DA: $p=0.009$; DOPAC: $p<0.01$; HVA: $p<0.05)$. The distinguished decrease in DA, DOPAC and HVA was found in 22MonR rats compared with 22Mon rats (DA: $p=0.009$; DOPAC: $p<0.01$; HVA: $p<0.01)$. There were no significant differences in the levels of DA, DOPAC and HVA between 22MonR-TP and 22MonR rats.

\section{TH and DAT mRNAs}

Group differences in TH mRNA (Figure 4A, $\chi^{2}=22.722$, $p<0.001$ ) and DAT mRNA (Figure 4B, $F_{(4,20)}=179.383$, $p<0.01)$ were detected in SN among 6Mon, 22Mon, 22Mon-TP, 22MonR and 22MonR-TP rats. Post hoc test found that both TH and DAT mRNAs were lower in 22Mon rats than in 6Mon rats (TH: $p=0.009$; DAT: $p<0.01)$. Compared with $22 \mathrm{Mon}$ rats, $\mathrm{TH}$ $(p=0.012)$ and DAT $(p<0.01)$ mRNAs significantly increased in 22Mon-TP rats. The reduced TH and DAT mRNAs were found in 22MonR rats compared with 22Mon rats (TH: $p=0.009$; DAT: $p<0.01)$ and in $22 \mathrm{MonR}-\mathrm{TP}$ rats compared with $22 \mathrm{MonR}$ rats (TH: $p=0.01$; DAT: $p<0.05$ ). Supplements of TP reduced TH and DAT at mRNA levels in SN of reserpine-treated aged male rats.
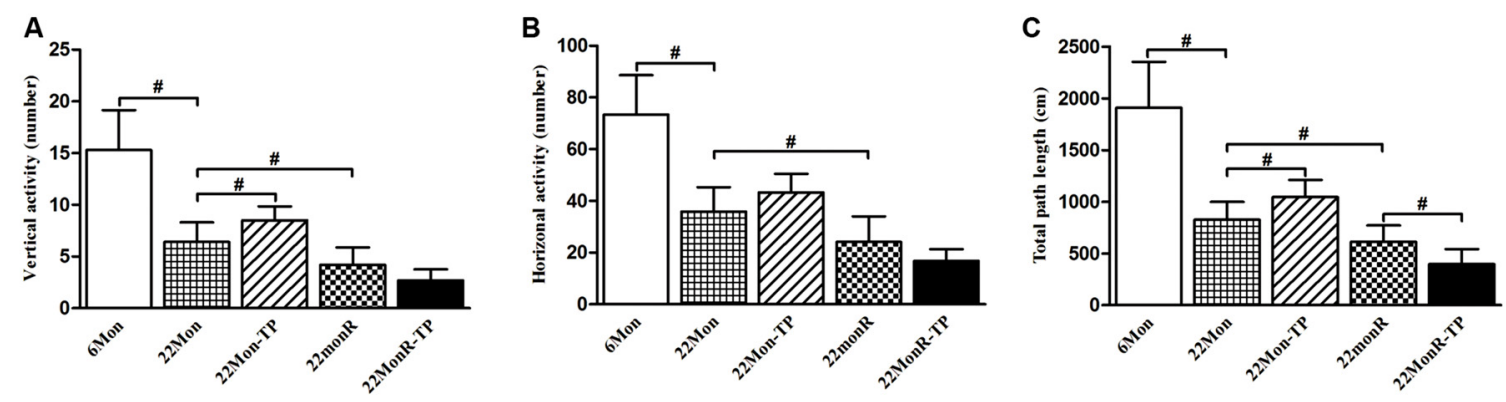

FIGURE 1 | Effects of testosterone propionate (TP) supplements on motor behaviors of reserpine-treated aged male rats. Each rat was individually examined by the open-field test. Open-field behavior (A: vertical activity, B: horizontal activity, C: the total path length) was recorded for 5 min and analyzed post hoc. Data were presented as mean $\pm \mathrm{SD}, n=10$. ${ }^{*} p<0.0125$ (Bonferroni correction). 

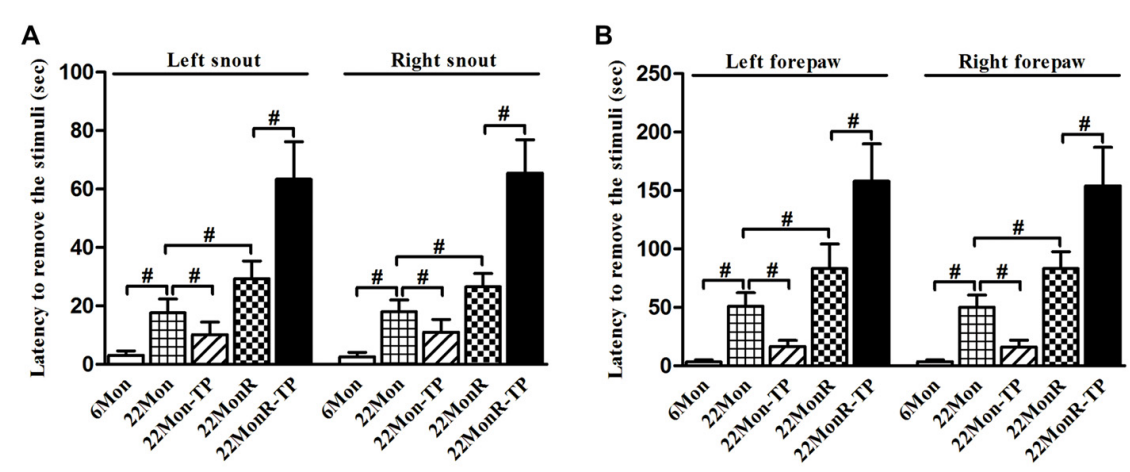

FIGURE 2 | Effects of TP supplements on the adhesive removal test of reserpine-treated aged male rats were examined. Latency to remove stimulus of adhesive paper from each side of snout (A) and each forepaw (B) was documented. Data were presented as mean $\pm \mathrm{SD}, n=10 .{ }^{\#} p<0.0125$ (Bonferroni correction).

\section{TH and DAT Proteins}

Western blot was used to analyze the levels of TH (Figure 4C) and DAT (Figure 4D) proteins in $\mathrm{SN}$ and in $\mathrm{CPu}$. TH and DAT were located at approximately 60 and $80 \mathrm{kDa}$, respectively. Group differences among 6Mon, 22Mon, 22Mon-TP, 22MonR and 22MonR-TP rats were detected in the levels of TH (Figure 4E, SN: $\left.F_{(4,20)}=129.438, p<0.01 ; \mathrm{CPu}: \chi^{2}=21.209, p<0.0001\right)$ and DAT (Figure 4F, SN: $F_{(4,20)}=122.01, p<0.01 ; \mathrm{CPu}$ : $\left.F_{(4,20)}=163.416, p<0.01\right)$ proteins. The post hoc test showed the prominent reduction of TH (SN: $p<0.01$; CPu: $p=0.009$ ) and DAT (SN: $p<0.01$; CPu: $p<0.01$ ) in SN and in CPu of $22 \mathrm{Mon}$ rats compared with $6 \mathrm{Mon}$ rats. Compared with $22 \mathrm{Mon}$ rats, the elevated TH (SN: $p<0.01$; CPu: $p=0.009$ ) and DAT (SN: $p<0.01$; CPu: $p<0.01$ ) were observed in 22Mon-TP rats and the decreased TH (SN: $p<0.05 ; \mathrm{CPu}: p=0.009)$ and DAT (SN: $p<0.05$; CPu: $p<0.01)$ were detected in 22MonR rats. In 22MonR-TP rats, TH (SN: $p<0.01$; CPu: $p=0.009$ ) and DAT (SN: $p<0.01$; CPu: $p<0.01$ ) were lower than in 22MonR rats. Supplements of TP diminished TH and DAT at protein levels in $\mathrm{SN}$ and $\mathrm{CPu}$ of reserpine-treated aged male rats.

\section{GSH and MDA}

To detect the effects of TP supplements on the levels of oxidative stress in reserpine-treated aged male rats, GSH and MDA, two important parameters of oxidative stress, were measured. Group differences among 6Mon, 22Mon, 22Mon-TP, 22MonR and 22MonR-TP rats were found in the levels of GSH (Figure 5A, $F_{(4,20)}=87.785, p<0.01$ ) and MDA (Figure 5B, $F_{(4,20)}=78.799$, $p<0.01)$ in SN. Post hoc test revealed the significant reduction of GSH $(p<0.01)$ and elevation of MDA $(p<0.01)$ in 22Mon rats compared with 6 Mon rats. The increased GSH $(p<0.05)$ and decreased MDA $(p<0.01)$ were detected in 22Mon-TP rats and the reduced GSH $(p<0.01)$ and elevated MDA $(p<0.01)$ were observed in $22 \mathrm{MonR}$ rats, compared with $22 \mathrm{Mon}$ rats. Supplement of TP caused the significant reduction of GSH $(p<0.01)$ and elevation of MDA $(p<0.01)$ in 22MonR-TP rats. Supplements of TP aggravated the oxidative stress in $\mathrm{SN}$ of reserpine-treated aged male rats.

\section{Nrf2-ARE Pathway}

To evaluate the effects of TP supplements on Nrf2-ARE pathway in $\mathrm{SN}$ of reserpine-treated aged male rats, Nrf2, $\mathrm{HO}-1$ and NQO1 were examined at mRNA and protein levels.

\section{Nrf2, HO-1 and NQO1 mRNAs}

Group differences among 6Mon, 22Mon, 22Mon-TP, 22MonR and 22MonR-TP rats were found in Nrf2 mRNA (Figure 6A, $F_{(4,20)}=274.248, p<0.01$ ), HO-1 mRNA (Figure 6B,
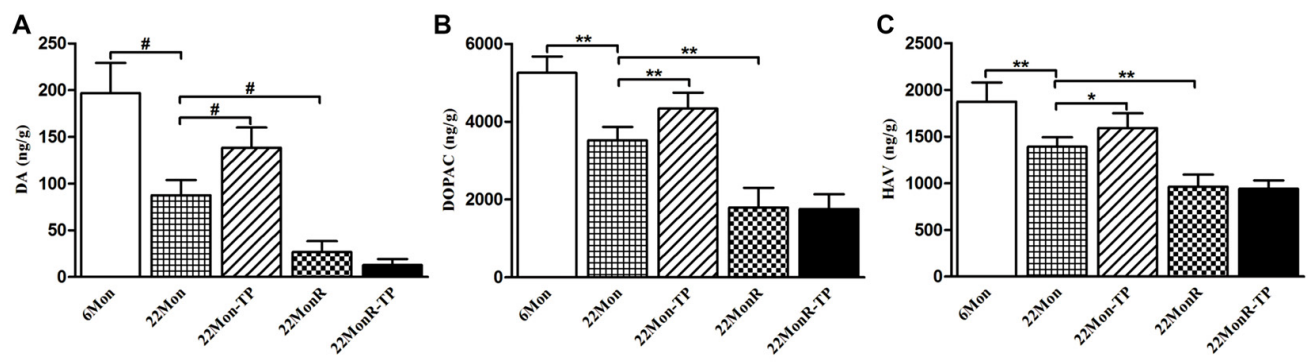

FIGURE 3 | Effects of TP supplements on dopamine (DA) and its metabolites in caudate putamen (CPu) of reserpine-treated aged male rats. DA (A), 3,4-dihydroxyphenylacetic acid (DOPAC; B) and homovanillic acid (HVA; C) in CPu of rats in each group were detected by LC-MS/MS assay. Data were presented as mean $\pm \mathrm{SD}, n=5$. ${ }^{\#} p<0.0125$ (Bonferroni correction); ${ }^{*} p<0.05,{ }^{* *} p<0.01$. 


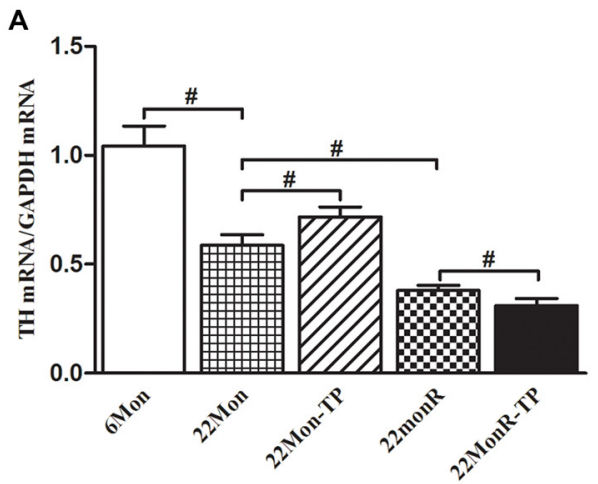

C

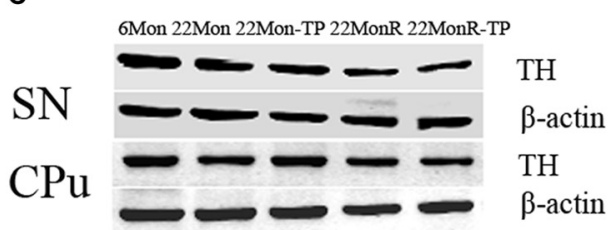

E

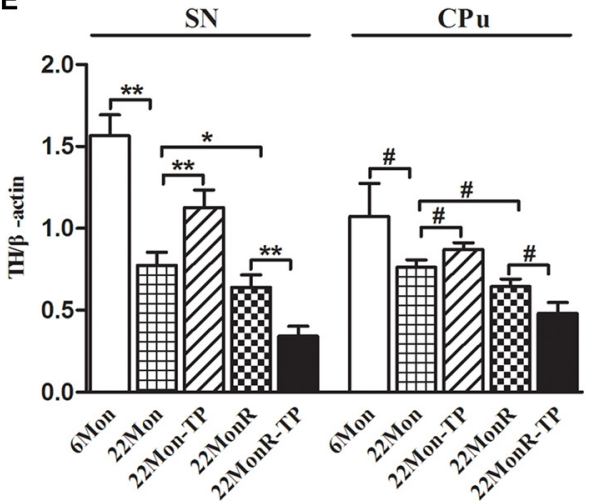

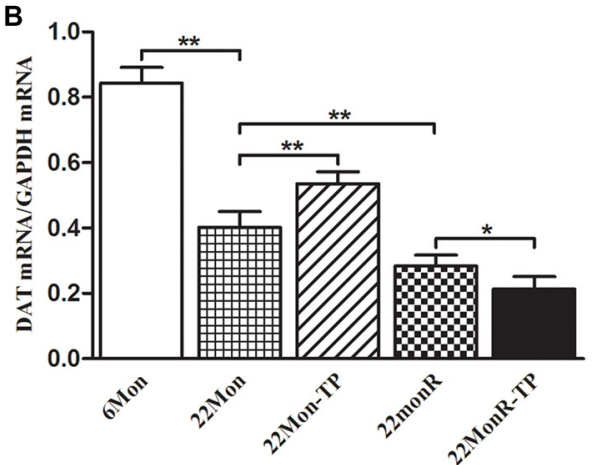

D

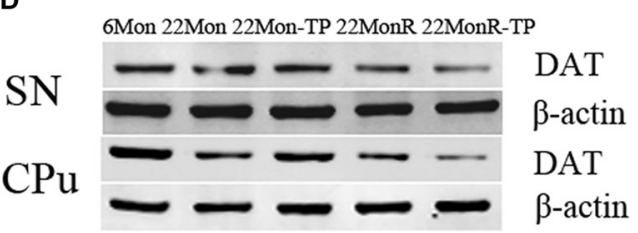

$\mathbf{F}$

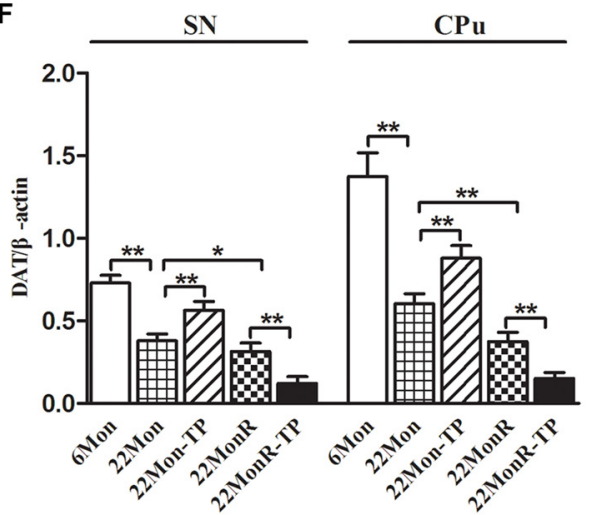

FIGURE 4 | Effects of TP supplements on tyrosine hydroxylase (TH) and DA transporter (DAT) in substantia nigra (SN) and CPu of reserpine-treated aged male rats. TH (A) and DAT (B) mRNAs were examined by quantitative real-time PCR. TH $(\mathbf{C}, \mathbf{E})$ and DAT $(\mathbf{D}, \mathbf{F})$ proteins were detected by Western blot. Data were presented as mean $\pm \mathrm{SD}, n=5$. ${ }^{*} p<0.0125$ (Bonferroni correction); ${ }^{*} p<0.05$, ${ }^{* *} p<0.01$.

$\chi^{2}=22.900, p<0.001$ ) and NQO1 mRNA (Figure 6C, $\left.F_{(4,20)}=115.038, p<0.01\right)$ in SN. Post hoc test showed that Nrf2, HO-1 and NQO1 mRNAs in SN significantly reduced in 22Mon rats compared with $6 \mathrm{Mon}$ rats $(\mathrm{Nrf} 2: p<0.01$; HO- $1: p=0.009$; NQO1: $p<0.01$ ) and with 22Mon-TP rats (Nrf2: $p<0.01$; HO-1: $p=0.009$; NQO1: $p<0.01$ ). The marked reduction of Nrf2, HO-1 and NQO1 mRNAs were observed in 22MonR rats compared with 22Mon rats (Nrf2: $p<0.01$; HO-1: $p=0.009$; NQO1: $p<0.01)$ and in 22MonR-TP rats compared with 22MonR rats (Nrf2: $p<0.01$;O- $1: p=0.009$; NQO1: $p<0.01$ ). Supplements of TP reduced Nrf2, HO-1 and NQO1 at mRNA levels in SN of reserpine-treated aged male rats.

\section{Nrf2, HO-1 and NQO1 Proteins}

Group differences of the Nrf2 (110 kDa), HO-1 (32 kDa) and NQO1 $(31 \mathrm{kDa})$ proteins in SN (Figure 6D) among 6Mon, 22Mon, 22Mon-TP, 22MonR and 22MonR-TP rats were found
(Figure 6E, Nrf2: $F_{(4,20)}=54.592, p<0.01$; Figure 6F, HO-1: $F_{(4,20)}=77.030, p<0.01$; Figure 6G, NQO1: $F_{(4,20)}=139.64$, $p<0.01)$. Post hoc test revealed that Nrf2, HO-1 and NQO1 proteins significantly reduced in 22Mon rats compared with 6 Mon rats $(p<0.01)$ and with 22Mon-TP rats $(p<0.01)$. Nrf2, HO-1 and NQO1 proteins were found lower in $22 \mathrm{MonR}$ rats than in 22Mon rats (Nrf2: $p<0.05$; HO- $1: p<0.05$; NQO1: $p<0.01)$ and in $22 \mathrm{MonR}-\mathrm{TP}$ rats than in 22MonR rats (Nrf2: $p<0.01$; HO-1: $p<0.01$; NQO1: $p<0.05)$. Supplements of $\mathrm{TP}$ reduced Nrf2, HO-1 and NQO1 at protein levels in $\mathrm{SN}$ of reserpine-treated aged male rats.

\section{DISCUSSION}

The present studies showed that TP supplements to aged male rats had beneficial effects on NSDA system and DA-related behaviors. The reduced DA and its metabolites, as well as the 

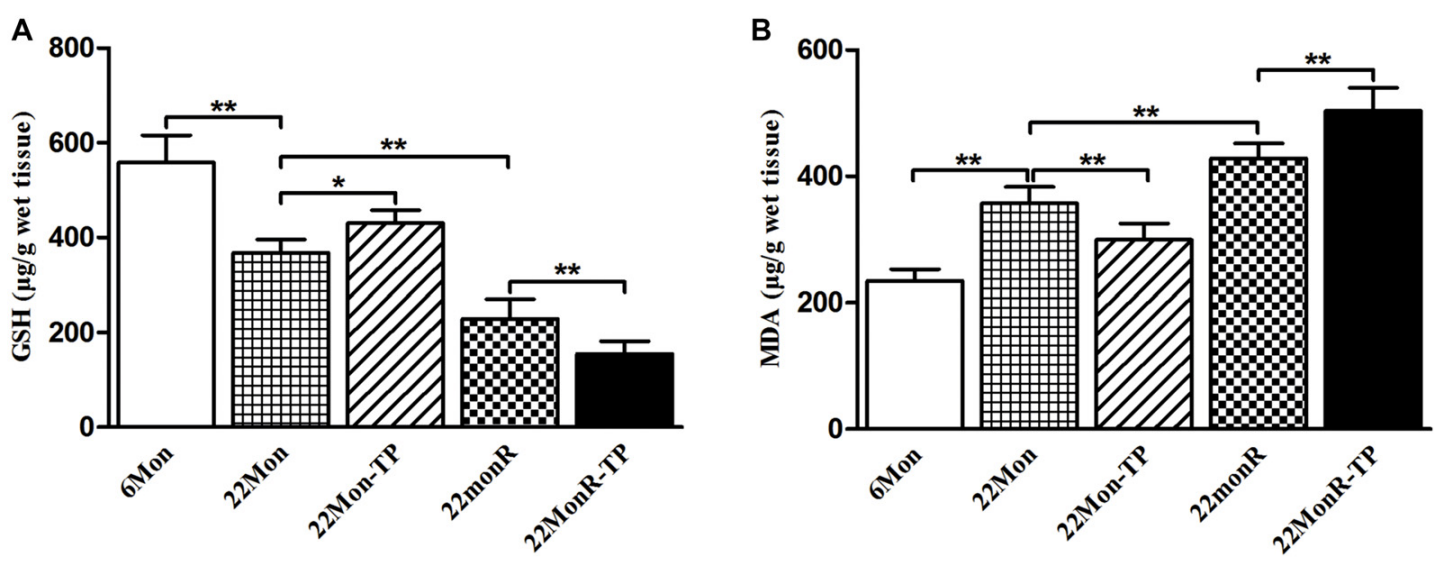

FIGURE 5 | Effects of TP supplements on the levels of oxidative stress in SN of reserpine-treated aged male rats. The levels of glutathione (GSH; A) and malondialdehyde (MDA; B) were examined spectrophotometrically. Data were presented as mean $\pm \mathrm{SD}, n=5 .{ }^{*} p<0.05,{ }^{* *} p<0.01$.

decreased TH and DAT in NSDA system of aged male rats were significantly ameliorated by TP supplements. Administration of TP to aged male rats enhanced the expression of $\mathrm{Nrf} 2$, HO-1 and NQO1 in SN. However, the beneficial effects of TP supplements on NSDA system and DA-related behaviors in aged male rats were reversed by reserpine pretreatment to them. Reserpine treatment induced the severe oxidative stress and downregulated the expressions Nrf2, HO-1 and NQO1 in the $\mathrm{SN}$ of aged male rats. Supplements of TP exacerbated the defects in NSDA system and DA-related behaviors, as well as oxidative damages in reserpine-treated aged male rats. The present results suggested that the efficacy of TP supplements on impaired NSDA system was related to the status of oxidative stress regulated by Nrf2-ARE pathway in experimental rats.

Oxidative stress is one of the important factors implicated in the dopaminergic dysfunctions (Chen et al., 2008; Hwang, 2013). With advancing age, dopaminergic neurons undergo more oxidative stress, as DA auto-oxidation increases markedly with aging (Fornstedt, 1990; Fornstedt et al., 1990; Kumar et al., 2012). Consistent with our previous studies (Cui et al., 2012; Wang et al., 2016; Zhang et al., 2016), the present studies showed the motor deficits, NSDA dysfunctions and oxidative damages in aged male rats, as well as the amelioratory effects of TP administration on them and the involvement of Nrf2-ARE pathway in amelioratory effects of TP supplements in aged male rats (Zhang et al., 2016). However, androgens may not always be neuroprotective (Gavrielides et al., 2006; Cunningham et al., 2009). The different effects of TP supplements to the aged experimental animals were found in the present studies. Supplements of TP ameliorated the behavioral deficits and defects of NSDA system in natural aged male rats, but the same treatments aggravated the deficits in behaviors and in NSDA system in reserpine-treated aged male rats. The status of oxidative stress in them might be critical in determining whether TP plays a neuroprotective or neurotoxic role as suggested by Holmes et al. (2013) in vitro studies. Testosterone protects dopaminergic N27 cells from subsequent oxidative insults in a low oxidative stress environment and exacerbates oxidative damages to the cultured cells in high oxidative stress milieus (Holmes et al., 2013). Furthermore, when androgen was used prior to coming oxidative insults, it exerted neuroprotective effects (Cheng et al., 2009; Uchida et al., 2009; Persky et al., 2013; Toro-Urrego et al., 2016). Testosterone protects astrocytic cells against glucose deprivation (Toro-Urrego et al., 2016) and reduces infarction in model of focal ischemia in male rats and male mice (Cheng et al., 2009; Uchida et al., 2009). In the present studies, although the reduced GSH and elevated MDA were observed in 22Mon rats compared with 6 Mon rats, which indicated the occurrence of oxidative stress in natural aging rats, much lower GSH and higher MDA was detected in 22MonR rats compared with 22 Mon rats.

Reserpine, an oxidative stress inductor, aggravated the oxidative stress in $\mathrm{SN}$ of age male rats and reversed the amelioratory effects of TP on the deficits in behaviors and NSDA system of aged male rats. Reserpine alone decreased $\mathrm{GSH}$ and increased MDA in the SN of aged male rats. More oxidative damages were induced in the SN of reserpine-treated aged male rats compared with aged male rats. Similar to us, it is found that administration of reserpine to animals dramatically induces the alteration in the status of oxidative stress in $\mathrm{CPu}$ (Spina and Cohen, 1989; Bilska et al., 2007), a DA-rich brain structure densely innervated by dopaminergic projection arising from the SN pars compacta. A considerable rise in the levels of oxidized GSH was detected in $\mathrm{CPu}$ of reserpine-treated adult rats (Bilska et al., 2007) and mice (Spina and Cohen, 1989). Pretreatment of androgens promotes the expression of different components of the antioxidant defense systems (Ahlbom et al., 1999; Toro-Urrego et al., 2016) to resist the coming oxidative injuries. Under the physiology condition, Nrf2 is sequestered in the cytoplasm by the repressor protein Keap1. However, under conditions of stress, Nrf2 is released from Keap1 and translocates to the nucleus (Carmona-Aparicio et al., 2015). Subsequently, Nrf2 binds to ARE to activate the transcription of corresponding downstream antioxidant genes, such as HO-1 and NQO1 (Prestera et al., 1995; Lee and Johnson, 2004; Dou et al., 2016) to 

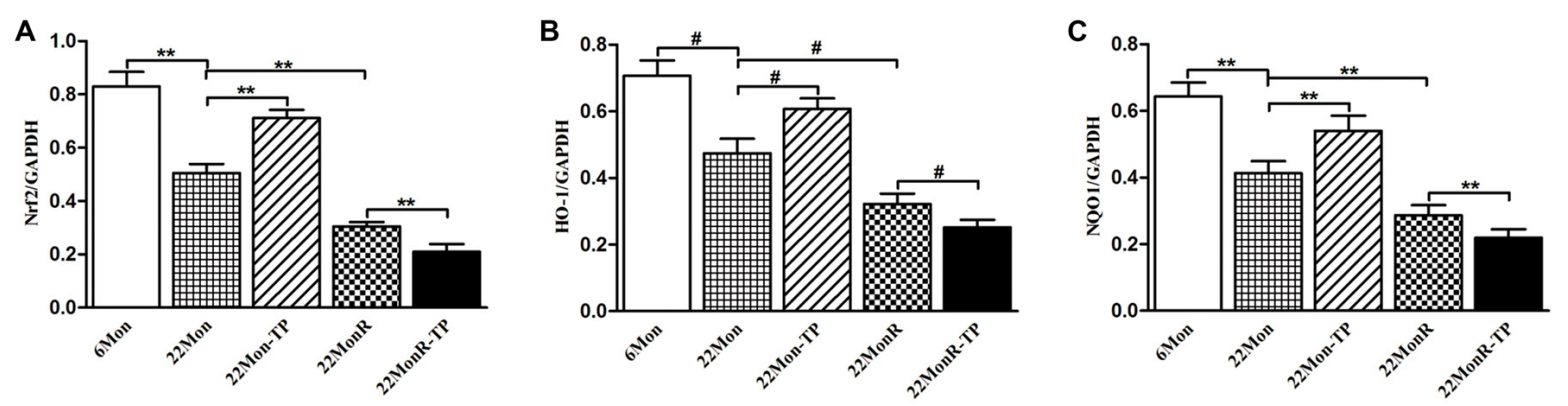

D
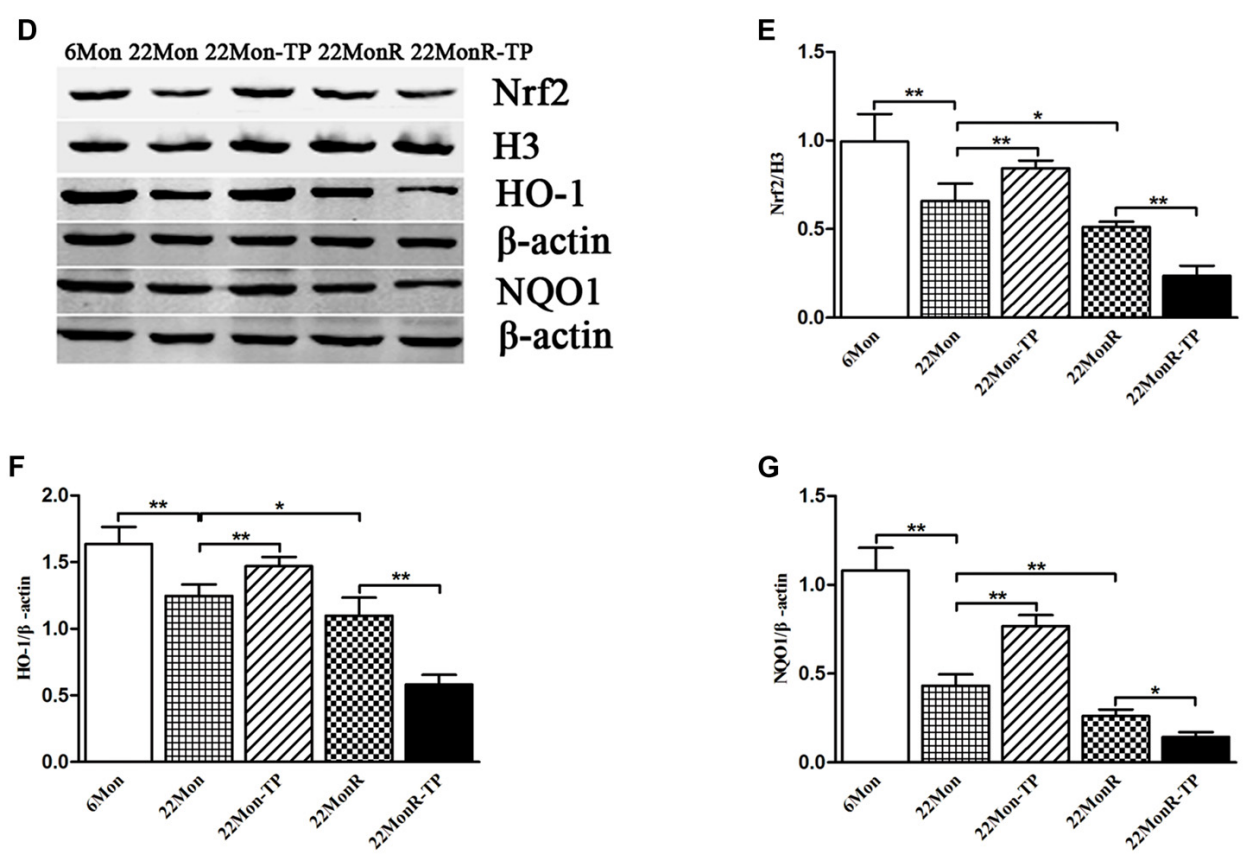

FIGURE 6 | Effects of TP supplements on nuclear factor erythroid 2-related factor 2 (Nrf2), heme oxygenase-1 (HO-1) and NAD(P)H:quinone oxidoreductase-1 (NQO1) in SN of reserpine-treated aged male rats. Nrf2 (A), HO-1 (B) and NQO1 (C) mRNAs were revealed by quantitative real-time PCR. Nrf2 (D,E), HO-1 (D,F) and NQ01 (D,G) proteins were detected by Western blot. Data were presented as mean $\pm \mathrm{SD}, n=5$. ${ }^{*} p<0.0125$ (Bonferroni correction); ${ }^{*} p<0.05,{ }^{* *} p<0.01$.

suppress the oxidative stress and maintain the redox balance. In the present studies, we found TP supplements to natural aged male rats at 21-month old upregulated the expression of Nrf2, HO-1 and NQO1 in the SN. Activation of Nrf2-ARE pathway protects neurons against oxidative and excitotoxic damages (Ahmad et al., 2006; Chen et al., 2011; Wang et al., 2014; Carmona-Aparicio et al., 2015). Disruption of Nrf2-ARE pathway results in an increased susceptibility to oxidative insults and other toxicants (Kwak et al., 2002). Significant reduction in the expressions of Nrf2, HO-1 and NQO1 was detected in reserpine-treated aged male rats, which indicated the aggravated antioxidative capability. Thus, the exacerbation effects of TP supplements on the deficits of NSDA system in reserpine-treated aged male rats might be related to the increased oxidative stress due to reserpine-downregulating Nrf2-ARE pathway in some extent.

In the present studies, only reserpine was chosen as oxidative stress inductor. More methodology for inducing oxidative stress should be employed to testify the potential influence of oxidative stress on testosterone supplements in the following studies. Furthermore, contrary to our finding, reserpine upregulates Nrf2 epigenetically in mouse skin epidermal JB6 P+ cells (Hong et al., 2016). The following reason might explain the response differences to reserpine. It is found that Nrf2 differs between various neuronal subpopulations and regulates different gene products, such as in the hippocampal neurons vs. nigral neurons (Kraft et al., 2004). The similar situation might occur in nigral neurons vs. mouse skin epidermal JB6 P+ cells. Whether it is true or not is necessary to be investigated.

In conclusion, TP supplements enhanced the activity of NSDA system and ameliorated the motor deficits of aged male rats. Reserpine pretreatment induced the elevated oxidative stress, downregulated Nrf2 expressions and reversed the amelioratory effects of TP supplements on the deficits in NSDA system of aged male rats. The status of oxidative stress might 
determine the efficacy of testosterone supplements to aged male rats.

\section{AUTHOR CONTRIBUTIONS}

RC and YK performed PCR, western blot experiments, analyzed the data and wrote the manuscript. LW and SL performed the biochemical experiments and LC-MS/MS assay and contributed to drafting. XJ, WY and GZ carried out behavioral experiments and the measurement of serum testosterone levels. HC interpreted data and revised the manuscript. GS designed the study, analyzed the data and revised the manuscript.

\section{REFERENCES}

Abreu, P., Hernandez, G., Calzadilla, C. H., and Alonso, R. (1988). Reproductive hormones control striatal tyrosine hydroxylase activity in the male rat. Neurosci. Lett. 95, 213-217. doi: 10.1016/0304-3940(88)90659-3

Ahlbom, E., Grandison, L., Bonfoco, E., Zhivotovsky, B., and Ceccatelli, S. (1999). Androgen treatment of neonatal rats decreases susceptibility of cerebellar granule neurons to oxidative stress in vitro. Eur. J. Neurosci. 11, 1285-1291. doi: 10.1046/j.1460-9568.1999.00529.x

Ahmad, A. S., Zhuang, H., and Doré, S. (2006). Heme oxygenase-1 protects brain from acute excitotoxicity. Neuroscience 141, 1703-1708. doi: 10.1016/j. neuroscience.2006.05.035

Bilska, A., Dubiel, M., Sokołowska-Jezewicz, M., Lorenc-Koci, E., and Włodek, L. (2007). $\alpha$-lipoic acid differently affects the reserpine-induced oxidative stress in the striatum and prefrontal cortex of rat brain. Neuroscience 146, 1758-1771. doi: 10.1016/j.neuroscience.2007.04.002

Borbélyová, V., Domonkos, E., Bábíčková, J., Tóthová, L., Bosý, M., Hodosy, J., et al. (2016). No effect of testosterone on behavior in aged Wistar rats. Aging 8, 2848-2861. doi: 10.18632/aging. 101096

Carmona-Aparicio, L., Pérez-Cruz, C., Zavala-Tecuapetla, C., Granados-Rojas, L., Rivera-Espinosa, L., Montesinos-Correa, H., et al. (2015). Overview of Nrf2 as therapeutic target in epilepsy. Int. J. Mol. Sci. 16, 18348-18367. doi: 10.3390/ijms160818348

Chen, L., Ding, Y., Cagniard, B., Van Laar, A. D., Mortimer, A., Chi, W., et al. (2008). Unregulated cytosolic dopamine causes neurodegeneration associated with oxidative stress in mice. J. Neurosci. 28, 425-433. doi: 10.1523/JNEUROSCI.3602-07.2008

Chen, G., Fang, Q., Zhang, J., Zhou, D., and Wang, Z. (2011). Role of the Nrf2-ARE pathway in early brain injury after experimental subarachnoid hemorrhage. J. Neurosci. Res. 89, 515-523. doi: 10.1002/jnr.22577

Chen, R., Osterhaus, G., McKerchar, T., and Fowler, S. C. (2003). The role of exogenous testosterone in cocaine-induced behavioral sensitization and plasmalemmal or vesicular dopamine uptake in castrated rats. Neurosci. Lett. 351, 161-164. doi: 10.1016/s0304-3940(03)00975-3

Cheng, J., Hu, W., Toung, T. J., Zhang, Z., Parker, S. M., Roselli, C. E., et al. (2009). Age-dependent effects of testosterone in experimental stroke. J. Cereb. Blood Flow Metab. 29, 486-494. doi: 10.1038/jcbfm.2008.138

Cui, R., Zhang, G., Kang, Y., Cheng, Q., Tan, H., Cui, H., et al. (2012). Amelioratory effects of testosterone propionate supplement on behavioral, biochemical and morphological parameters in aged rats. Exp. Gerontol. 47, 67-76. doi: 10.1016/j.exger.2011.10.009

Cunningham, R. L., Giuffrida, A., and Roberts, J. L. (2009). Androgens induce dopaminergic neurotoxicity via caspase-3-dependent activation of protein kinase C 8 . Endocrinology 150, 5539-5548. doi: 10.1210/en.2009-0640

Cunningham, G. R., and Toma, S. M. (2011). Clinical review: why is androgen replacement in males controversial? J. Clin. Endocrinol. Metab. 96, 38-52. doi: $10.1210 /$ jc.2010-0266

de Souza Silva, M. A., Mattern, C., Topic, B., Buddenberg, T. E., and Huston, J. P. (2009). Dopaminergic and serotonergic activity in neostriatum and nucleus accumbens enhanced by intranasal administration of testosterone. Eur. Neuropsychopharmacol. 19, 53-63. doi: 10.1016/j.euroneuro.2008.08.003
All authors have read and approved the final version of the manuscript.

\section{FUNDING}

This project was financially supported in part by the Natural Science Foundation of China (No. 81200252), the Natural Science Foundation of Hebei Province of China (No. C2014206283 and No. C2017206072), the Foundation of the Educational Commission of Hebei Province of China (No. QN20131096) and the Research Development Fund of Hebei Medical University (No. kyfz023).

Dluzen, D. E., and Ramirez, V. D. (1989). Effects of orchidectomy on nigro-striatal dopaminergic function: behavioral and physiological evidence. J. Neuroendocrinol. 1, 285-290. doi: 10.1111/j.1365-2826.1989. tb00117.x

Dou, T., Yan, M., Wang, X., Lu, W., Zhao, L., Lou, D., et al. (2016). Nrf2/ARE pathway involved in oxidative stress induced by Paraquat in human neural progenitor cells. Oxid. Med. Cell. Longev. 2016:8923860. doi: $10.1155 / 2016 / 8923860$

Estrada, M., Varshney, A., and Ehrlich, B. E. (2006). Elevated testosterone induces apoptosis in neuronal cells. J. Biol. Chem. 281, 25492-25501. doi: 10.1074/jbc. M603193200

Fornstedt, B. (1990). Role of catechol autooxidation in the degeneration of dopamine neurons. Acta Neurol. Scand. Suppl. 129, 12-14. doi: 10.1111/j.16000404.1990.tb02595.x

Fornstedt, B., Pileblad, E., and Carlsson, A. (1990). In vivo autoxidation of dopamine in guinea pig striatum increases with age. J. Neurochem. 55, 655-659. doi: 10.1111/j.1471-4159.1990.tb04183.x

Frye, C. A., Edinger, K. L., Lephart, E. D., and Walf, A. A. (2010). $3 \alpha-$ androstanediol, but not testosterone, attenuates age-related decrements in cognitive, anxiety, and depressive behavior of male rats. Front. Aging Neurosci. 2:15. doi: 10.3389/fnagi.2010.00015

Frye, C. A., Edinger, K., and Sumida, K. (2008). Androgen administration to aged male mice increases anti-anxiety behavior and enhances cognitive performance. Neuropsychopharmacology 33, 1049-1061. doi: 10.1038/sj.npp. 1301498

Fuentes, P., Paris, I., Nassif, M., Caviedes, P., and Segura-Aguilar, S. (2007). Inhibition of VMAT-2 and DT-diaphorase induced cell death in a substantia nigra-derived cell line-an experimental cell model for dopamine toxicity studies. Chem. Res. Toxicol. 20, 776-783. doi: 10.1021/tx600325u

Gavrielides, M. V., Gonzalez-Guerrico, A. M., Riobo, N. A., and Kazanietz, M. G. (2006). Androgens regulate protein kinase $\mathrm{C} \delta$ transcription and modulate its apoptotic function in prostate cancer cells. Cancer Res. 66, 11792-11801. doi: 10.1158/0008-5472.can-06-1139

Hamilton, M. L., Van Remmen, H., Drake, J. A., Yang, H., Guo, Z. M., Kewitt, K. et al. (2001). Does oxidative damage to DNA increase with age? Proc. Natl. Acad. Sci. U S A 98, 10469-10474. doi: 10.1073/pnas.171202698

Hernandez, L., Gonzalez, L., Murzi, E., Páez, X., Gottberg, E., and Baptista, T. (1994). Testosterone modulates mesolimbic dopaminergic activity in male rats. Neurosci. Lett. 171, 172-174. doi: 10.1016/0304-3940(94)90632-7

Hollander, M., Koudstaal, P. J., Bots, M. L., Grobbee, D. E., Hofman, A., and Breteler, M. M. (2003). Incidence, risk, and case fatality of first ever stroke in the elderly population. The Rotterdam Study. J. Neurol. Neurosurg. Psychiatry. 74, 317-321. doi: 10.1136/jnnp.74.3.317

Holmes, S., Abbassi, B., Su, C., Singh, M., and Cunningham, R. L. (2013). Oxidative stress defines the neuroprotective or neurotoxic properties of androgens in immortalized female rat dopaminergic neuronal cells. Endocrinology 154, 4281-4292. doi: 10.1210/en.2013-1242

Hong, B., Su, Z., Zhang, C., Yang, Y., Guo, Y., Li, W., et al. (2016). Reserpine inhibit the JB6 $\mathrm{P}+$ cell transformation through epigenetic reactivation of Nrf2-mediated anti-oxidative stress pathway. AAPS J. 18, 659-669. doi: 10.1208/s12248-016-9901-6 
Hwang, O. (2013). Role of oxidative stress in Parkinson's disease. Exp. Neurobiol. 22, 11-17. doi: 10.5607/en.2013.22.1.11

Kindlundh, A. M., Rahman, S., Lindblom, J., and Nyberg, F. (2004). Increased dopamine transporter density in the male rat brain following chronic nandrolone decanoate administration. Neurosci. Lett. 356, 131-134. doi: 10.1016/j.neulet.2003.11.040

Kraft, A. D., Johnson, D. A., and Johnson, J. A. (2004). Nuclear factor E2-related factor 2-dependent antioxidant response element activation by tert-butylhydroquinone and sulforaphane occurring preferentially in astrocytes conditions neurons against oxidative insult. J. Neurosci. 24, 1101-1112. doi: 10.1523/JNEUROSCI.3817-03.2004

Kregel, K. C., and Zhang, H. J. (2007). An integrated view of oxidative stress in aging: basic mechanisms, functional effects, and pathological considerations. Am. J. Physiol. Regul. Integr. Comp. Physiol. 292, R18-R36. doi: 10.1152/ajpregu.00327.2006

Kumar, H., Lim, H. W., More, S. V., Kim, B. W., Koppula, S., Kim, I. S., et al. (2012). The role of free radicals in the aging brain and Parkinson's disease: convergence and parallelism. Int. J. Mol. Sci. 13, 10478-10504. doi: 10.3390/ijms130810478

Kwak, M. K., Itoh, K., Yamamoto, M., and Kensler, T. W. (2002). Enhanced expression of the transcription factor Nrf2 by cancer chemopreventive agents: role of antioxidant response element-like sequences in the nrf2 promoter. Mol. Cell. Biol. 22, 2883-2892. doi: 10.1128/mcb.22.9.2883-2892.2002

Lee, J., and Johnson, J. A. (2004). An important role of Nrf2-ARE pathway in cellular defense mechanism. J. Biochem. Mol. Biol. 37, 139-143. doi: $10.5483 /$ bmbrep.2004.37.2.139

Lohr, J. B. (1991). Oxygen free radicals and neuropsychiatric illness. Arch. Gen. Psychiatry 48, 1097-1106. doi: 10.1001/archpsyc.1991.01810360061009

Martínez-Sanchis, S., Aragon, C. M. G., and Salvador, A. (2002). Cocaine-induced locomotor activity is enhanced by exogenous testosterone. Physiol. Behav. 76, 605-609. doi: 10.1016/s0031-9384(02)00764-3

Metzger, R. R., Brown, J. M., Sandoval, V., Rau, K. S., Elwan, M. A., Miller, G. W., et al. (2002). Inhibitory effect of reserpine on dopamine transporter function. Eur. J. Pharmacol. 456, 39-43. doi: 10.1016/s0014-2999(02)02647-x

Meydan, S., Kus, I., Tas, U., Ogeturk, M., Sancakdar, E., Dabak, D. O., et al. (2010). Effects of testosterone on orchiectomy-induced oxidative damage in the rat hippocampus. J. Chem. Neuroanat. 40, 281-285. doi: 10.1016/j.jchemneu. 2010.07.006

Miller, D. M., Singh, I. N., Wang, J. A., and Hall, E. D. (2015). Nrf2-ARE activator carnosic acid decreases mitochondrial dysfunction, oxidative damage and neuronal cytoskeletal degradation following traumatic brain injury in mice. Exp. Neurol. 264, 103-110. doi: 10.1016/j.expneurol.2014.11.008

Mitchell, E., Thomas, D., and Burnet, R. (2006). Testosterone improves motor function in Parkinson's disease. J. Clin. Neurosci. 13, 133-136. doi: 10.1016/j. jocn.2005.02.014

Okun, M. S., Walter, B. L., McDonald, W. M., Tenover, J. L., Green, J., Juncos, J. L., et al. (2002). Beneficial effects of testosterone replacement for the nonmotor symptoms of Parkinson disease. Arch. Neurol. 59, 1750-1753. doi: 10.1001/archneur.59.11.1750

Paris, I., Dagnino-Subiabre, A., Marcelain, K., Bennett, L. B., Caviedes, P., Caviedes, R., et al. (2001). Copper neurotoxicity is dependent on dopaminemediated copper uptake and one-electron reduction of aminochrome in a rat substantia nigra neuronal cell line. J. Neurochem. 77, 519-529. doi: 10.1046/j. 1471-4159.2001.00243.x

Paxinos, G., and Watson, C. (2007). The Rat Brain in Stereotaxic Coordinates, 6th Edn. Orlando, FL: Academic Press.

Persky, R. W., Liu, F., Xu, Y., Weston, G., Levy, S., Roselli, C. E., et al. (2013). Neonatal testosterone exposure protects adult male rats from stroke. Neuroendocrinology 97, 271-282. doi: 10.1159/000343804

Pike, C. J., Carroll, J. C., Rosario, E. R., and Barron, A. M. (2009). Protective actions of sex steroid hormones in Alzheimer's disease. Front. Neuroendocrinol. 30, 239-258. doi: 10.1016/j.yfrne.2009.04.015

Prestera, T., Talalay, P., Alam, J., Ahn, Y. I., Lee, P. J., and Choi, A. M. (1995). Parallel induction of heme oxygenase- 1 and chemoprotective phase 2 enzymes by electrophiles and antioxidants: regulation by upstream antioxidantresponsive elements (ARE). Mol. Med. 1, 827-837.

Schallert, T., Upchurch, M., Lobaugh, N., Farrar, S. B., Spirduso, W. W., Gilliam, P., et al. (1982). Tactile extinction: distinguishing between sensorimotor and motor asymmetries in rats with unilateral nigrostriatal damage. Pharmacol. Biochem. Behav. 16, 455-462. doi: 10.1016/00913057(82)90452-x

Spina, M. B., and Cohen, G. (1989). Dopamine turnover and glutathione oxidation: implications for Parkinson disease. Proc. Natl. Acad. Sci. U S A 86, 1389-1400. doi: 10.1073/pnas.86.4.1398

Spritzer, M. D., Daviau, E. D., Coneeny, M. K., Engelman, S. M., Prince, W. T., and Rodriguez-Wisdom, K. N. (2011). Effects of testosterone on spatial learning and memory in adult male rats. Horm. Behav. 59, 484-496. doi: 10.1016/j. yhbeh.2011.01.009

Sun, D., Dong, L., Guo, P., Shi, X., Gao, J., Ren, Y., et al. (2013). Simultaneous detection of flavonoids and phenolic acids in herba lysimachiae and herba desmodii styracifolii using liquid chromatography tandemmass spectrometry. Food Chem. 138, 139-147. doi: 10.1016/j.foodchem.2012.09.096

Tenover, J. S. (1992). Effects of testosterone supplementation in the aging male. J. Clin. Endocrinol. Metab. 75, 1092-1098. doi: 10.1210/jcem.75.4.1400877

Toro-Urrego, N., Garcia-Segura, L. M., Echeverria, V., and Barreto, G. E. (2016). Testosterone protects mitochondrial function and regulates neuroglobin expression in astrocytic cells exposed to glucose deprivation. Front. Aging Neurosci. 8:152. doi: 10.3389/fnagi.2016.00152

Uchida, M., Palmateer, J. M., Herson, P. S., DeVries, A. C., Cheng, J., and Hurn, P. D. (2009). Dose-dependent effects of androgens on outcome after focal cerebral ischemia in adult male mice. J. Cereb. Blood Flow Metab. 29, 1454-1462. doi: 10.1038/jcbfm.2009.60

van Muiswinkel, F. L., and Kuiperij, H. B. (2005). The Nrf2-ARE signalling pathway: promising drug target to combat oxidative stress in neurodegenerative disorders. Curr. Drug Targets CNS Neurol. Disord. 4, 267-281. doi: 10.2174/1568007054038238

Villar-Cheda, B., Dominguez-Meijide, A., Valenzuela, R., Granado, N., Moratalla, R., and Labandeira-Garcia, J. L. (2014). Aging-related dysregulation of dopamine and angiotensin receptor interaction. Neurobiol. Aging 35, 1726-1738. doi: 10.1016/j.neurobiolaging.2014.01.017

Wang, L., Kang, Y., Zhang, G., Zhang, Y., Cui, R., Yan, W., et al. (2016). Deficits in coordinated motor behavior and in nigrostriatal dopaminergic system ameliorated and VMAT2 expression up-regulated in aged male rats by administration of testosterone propionate. Exp. Gerontol. 78, 1-11. doi: $10.1016 /$ j.exger.2016.03.003

Wang, X., and Michaelis, E. K. (2010). Selective neuronal vulnerability to oxidative stress in the brain. Front. Aging Neurosci. 2:12. doi: 10.3389/fnagi.2010. 00012

Wang, W., Wu, Y., Zhang, G., Fang, H., Wang, H., Zang, H., et al. (2014). Activation of Nrf2-ARE signal pathway protects the brain from damage induced by epileptic seizure. Brain Res. 1544, 54-61. doi: 10.1016/j.brainres. 2013.12.004

Zhang, G., Li, S., Kang, Y., Che, J., Cui, R., Song, S., et al. (2016). Enhancement of dopaminergic activity and region-specific activation of Nrf2-ARE pathway by intranasal supplements of testosterone propionate in aged male rats. Horm. Behav. 80, 103-116. doi: 10.1016/j.yhbeh.2016.02.001

Zhang, G., Shi, G., Tan, H., Kang, Y., and Cui, H. (2011). Intranasal administration of testosterone increased immobile-sniffing, exploratory behavior, motor behavior and grooming behavior in rats. Horm. Behav. 59, 477-483. doi: 10.1016/j.yhbeh.2011.01.007

Zhang, G. L., Wang, W., Kang, Y. X., Xue, Y., Yang, H., Zhou, C. M., et al. (2013). Chronic testosterone propionate supplement could activated the Nrf2-ARE pathway in the brain and ameliorated the behaviors of aged rats. Behav. Brain Res. 252, 388-395. doi: 10.1016/j.bbr.2013.05.063

Conflict of Interest Statement: The authors declare that the research was conducted in the absence of any commercial or financial relationships that could be construed as a potential conflict of interest.

Copyright $\odot 2017$ Cui, Kang, Wang, Li, Ji, Yan, Zhang, Cui and Shi. This is an open-access article distributed under the terms of the Creative Commons Attribution License (CC BY). The use, distribution or reproduction in other forums is permitted, provided the original author(s) or licensor are credited and that the original publication in this journal is cited, in accordance with accepted academic practice. No use, distribution or reproduction is permitted which does not comply with these terms. 